\title{
Characterization of optically stimulated luminescence for assessment of breast doses in mammography screening
}

\author{
N. Alothmany ${ }^{1}$, N.I. Molla ${ }^{2}$, M. Yusuf ${ }^{3 \star}$, A. Saoudi ${ }^{4,5}$, N. Mail ${ }^{4,5}$, D. Alothmany ${ }^{3}$, M.A. Khafaji ${ }^{6}$, \\ H. Natto ${ }^{2}$, M. Tayeb ${ }^{2}$, F. Nadwi ${ }^{2}$, A. Jiman ${ }^{1}$ and A.A. Kinsara ${ }^{3}$ \\ 1 Electrical and Computer Engineering Department, Faculty of Engineering, King Abdulaziz University, Jeddah 21589, Saudi Arabia. \\ 2 Center of Radiation Protection and Training, King Abdulaziz University, Jeddah 21589, Saudi Arabia. \\ 3 Nuclear Engineering Department, Faculty of Engineering, King Abdulaziz University, Jeddah 21589, Saudi Arabia. \\ ${ }^{4}$ King Abdullah International Medical Research Center (KAIMRC), Jeddah, Saudi Arabia. \\ 5 Princess Norah Oncology Center (PNOC), National Guard Health Affairs (NGHA), Jeddah, Saudi Arabia. \\ ${ }^{6}$ Radiology Department, Faculty of Medicine, King Abdulaziz University, Jeddah 21859, Saudi Arabia.
}

Received 12 June 2015 - Accepted 17 December 2015

\begin{abstract}
Landauer optically stimulated luminescence (OSL) technology nanoDot dosimeters (OSLDs) are characterized for use in mammography screening at various tube voltages, $\mathrm{mAs}$ values and target/filter combinations. The average glandular dose (AGD) for a 50-mm breast, based on the representative compressed breast thickness of a 45-mm polymethyl methacrylate (PMMA) phantom, is assessed using OSLDs with different beam conditions. Further, the linearity of the OSLD response is measured and angular dependence tests are performed for various tube potentials, $\mathrm{mAs}$ and target/filter combinations. The breast-absorbed doses are measured at various depths for a $32-\mathrm{kVp} \mathrm{X}$-ray beam at $100 \mathrm{mAs}$, with a Mo/Rh target/filter combination. The measured incident air kerma values at different lateral positions exhibit a maximum deviation of $6 \%$, and the average relative response of the OSLDs at the reference point (center) with respect to various lateral positions is found to be $1.001 \pm 0.09 \%$. The calculated AGD values are in the $1.3 \pm 0.1-3.5 \pm 0.2 \mathrm{mGy}$ range, depending on the tube potential, tube loading and target/filter combinations. An exposure setup featuring the auto-exposure control (AEC) mode, $28 \mathrm{kVp}, 73.8 \mathrm{mAs}$, and a Mo/Rh target/filter combination may be preferred for mammography screening for a compressed breast thickness of $45 \mathrm{~mm}$.
\end{abstract}

Keywords: Optically Stimulated Luminescence Dosimeter (OSLD) / OSL characterization / mammography / breast phantom / Average Glandular Dose (AGD) / ionization chamber / kVp/mAs / target/filter

\section{Introduction}

Mammography is one of the procedures most widely used for the screening of small malignant lesions in the female breast, and its application has resulted in a significant reduction in breast cancer mortality, particularly among older women (Andersson, 1986; US Dpt of Health and Bassett, 1995; IAEA, 2007; Tsai et al., 2010). Breast cancer is considered to be the second largest cause of cancer deaths among female patients in many countries. Further, age is the most important single risk factor in the development of breast cancer, and the breast cancer incidence increases dramatically as a function of patient age (Dance et al., 1999, 2000; Young, 2002). However, early detection procedures using modern mammography systems with automatic beam quality selection and alternative target/filter combinations have led to approximately $15 \%$ reduction in average received dose values and up to $50 \%$ reduction in the received doses to large breasts, resulting in a significant decrease in the mortality rate (Poliei

\footnotetext{
^ myusufprajogo@hotmail.com
}

et al., 1987; Vinnicombe et al., 2009). The average glandular dose (AGD) received by glandular tissues in the female breast is considered to be the optimal indicator for estimating the potential cancer risk due to mammographic screening (Dance, 1990; Zoetelief et al., 1996). A significant increase in the breast screening AGD, over the IAEA recommended limit, which for a standard breast $(50 \mathrm{~mm}$ breast thickness and compressed breast thickness $45 \mathrm{~mm}$ ) is $2 \mathrm{mGy}$, is usually associated with a greater risk (Zoetelief et al., 1996). The AGD is determined via measurement of the incident air kerma $\left(K_{i}\right)$ on the entrance surface of the breast. In some studies, it is defined based on the entrance surface dose (ESD), the half-value layer (HVL) of the X-ray beam and the compressed breast thickness. The measured ESD includes contributions from backscattering, and must be corrected by incorporation of the backscatter factor (IAEA, 2007). Assumption of the conversion coefficient $C_{\text {DG50,Ki,PMMA }}(\mathrm{mGy} / \mathrm{mGy})$ is used to calculate the AGD for different breast thicknesses. This assumption is based on a breast composition of $50 \%$ fat and $50 \%$ glandular tissue (IAEA, 2007). In recent years, this conversion factor has 
been extended to incorporate a larger breast thickness interval and to account for other breast compositions and X-ray spectra (Dance et al., 2000; Oliveira et al., 2007).

Radiation dose is one of the aspects during quality assurance in producing images and the dosimeter is used for measuring the object dose from the source to assure that the dose received by the object is maintained as minimum as possible. There are several types of dosimeters are used for breast dosimetry. One type measures the optically stimulated luminescence (OSL) due to ionizing radiation exposure, in order to quantify the radiation dose absorbed by the target organ. Recently, OSL nanoDot dosimeters have been considered as potential mammography tools, in place of thermoluminescence dosimeters (TLDs) and metal-oxide semiconductor field-effect transistor (MOSFET)-based devices. TLDs have a tedious, complicated readout process of heating and annealing, and are time-consuming in comparison with other techniques, whereas their single-use restriction and higher cost are the greatest disadvantages of MOSFET dosimeters. The technology (MOSFET) in fact quantifies the damage to a semiconductor which is proportional to the absorbed radiation dose. The damage is irreversible, therefore the dosimeters have a limited lifetime that results in a recurring cost and single use. MOSFET dosimeters provide real-time mammography measurements but reproducibility of a single dosimeter measurement ranges from 15$31 \%$. Furthermore, OSL has better thermal stability compared with TLDs, longer dose storage lifetime compared with MOSFET dosimeters and the ability to be read multiple times with signal depletion less than $2 \%$, which could not be found in either TLDs or MOSFET dosimeters (Viamonte et al., 2008; Yusuf et al., 2014). The primary drawback with TLDs is their inability to provide the patient dose immediately postexposure. Moreover, OSL is extremely sensitive, 40-60 times the TL sensitivity of LiF:Mg,Ti. This permits the use of small samples of OSL material for dosimetric measurements. In these respects, OSL nanoDots are in an advantageous position. However, similar to other dosimeters, calibration of the OSL nanoDot is required before it can be used for radiation monitoring in diagnostic imaging as well as in personnel dosimetry. Previous studies on the use of OSL dosimeters (OSLDs) in computerized tomography (CT) dosimetry have indicated that OSLDs have potential use in measuring the absorbed radiation doses to the target organs relevant to diagnostic radiology ( Viamonte et al., 2008; Yukihara et al., 2008; Lavoie et al., 2011). The aim of the present work is to characterize OSLDs in various beam conditions.

\section{Materials and methods}

\subsection{OSLDs and readout system}

OSL technology is based on the assessment of light emissions. Radiation dose measurement via OSL is achieved using aluminum oxide powder reduced with carbon $\left(\mathrm{Al}_{2} \mathrm{O}_{3}\right.$ :C). Light-emitting diodes stimulate the $\mathrm{Al}_{2} \mathrm{O}_{3}: \mathrm{C}$ powder, which emits a light signal that is proportional to the absorbed radiation dose. The MicroStar ${ }^{\circledR}$ reader, developed by Landauer Inc., was first used for the dosimeter readout. The nanoDot

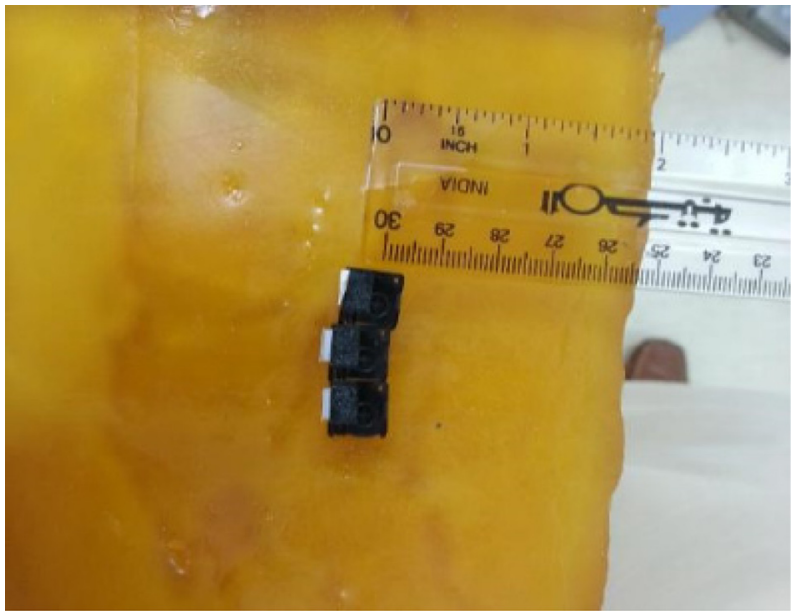

Figure 1. OSLD setup for calibration.

dosimeter used in this work was a dot of OSL material with a diameter of $4 \mathrm{~mm}$ and $0.2 \mathrm{~mm}$ thickness, enclosed in a $10 \times 10 \times 2 \mathrm{~mm}^{3}$ light-tight envelope, suitable for the detection of X-rays in the 5-40-keV range with minimum and maximum absorbed-dose detection limits of $0.05 \mathrm{mGy}$ and $10.0 \mathrm{~Gy}$, respectively.

\subsection{Calibration}

Selection of OSLD groups was based on annealing dozens of dosimeters from a single batch, quoted with batch sensitivity, reading out all of them in one session, considering the variation of background response within 3\% standard deviation, and then grouping them. Each dosimeter was used one time only either for single exposure or for simultaneous multiple exposures. For re-use, the dosimeters were annealed to the extent of background level counts, with 0.82 batch sensitivity, placed over a 45-mm mammography phantom (bolus) at a laterally centered position $50 \mathrm{~mm}$ from the chest edge wall, as shown in Figure 1. The OSLDs were irradiated three times with an X-ray beam with a tube potential of $28 \mathrm{kVp}$, 100-mAs tube loading and a Mo/Rh target/filter combination. The OSL response (counts) of each dosimeter was read out using a Landauer MicroStar ${ }^{\circledR}$ Inlight reader. The response was then adjusted for background correction, which was obtained from the average responses of control dosimeters. The radiation exposures were measured using a Secondary Standard Dosimetry Laboratory (SSDL)-level, 10X-6M dedicated mammography ion chamber with a $0.7-\mathrm{mg} / \mathrm{cm}^{2}$ metalized polyester window and $6-\mathrm{cm}^{3}$ active volume, coupled with an Accu-Pro dose-master system (model 9096) from RadCal Corporation. The quoted calibration accuracy of this chamber is $\pm 4 \%$ for $30-\mathrm{kVp}$ X-rays and 0.50-mm aluminum HVL, and the energy dependence response is $\pm 5 \%$ in the $10-40-\mathrm{keV}$ range. In the present work, an identical exposure situation was used for irradiation of the OSL nanoDot dosimeters and for measurements with the mammography chamber (Figure 2). 


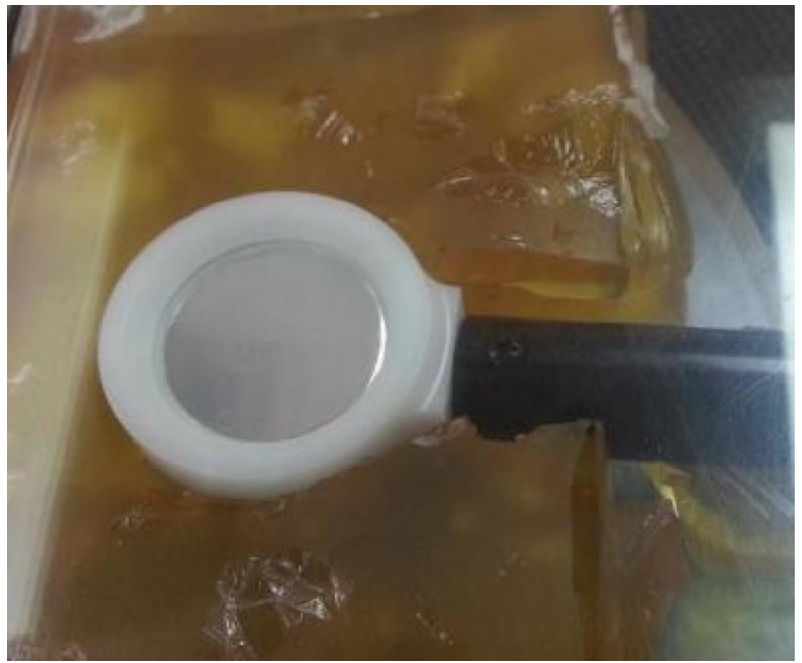

Figure 2. Ionization chamber setup for calibration.

The calibration factor was generated using the following equation:

$$
C F=\frac{\text { PMT Counts }(\text { Net })}{\text { Dose }(\text { mRad }) \times \text { Sensitivity }},
$$

where $C F$ is the OSLD calibration factor under measurement conditions, the photomultiplier (PMT) counts are the average of the net OSL nanoDot response, and the dose (mrad) is the ionization chamber reading, corrected for temperature and pressure. The values obtained from the ionization chamber readings were in the default unit mrad. All of them were converted to $\mathrm{mGy}$ units in the calculation of the average glandular dose (AGD). The ionization chamber reading was used for the reference dose during the experiment. "Sensitivity" refers to the batch sensitivity of the dosimeters.

\subsection{Reproducibility test of OSL nanoDot response}

This experiment was conducted to compare the reproducibility of the accumulated response of a set of OSLDs from multiple exposures with the sum of responses of another set of dosimeters, when each of them received a single exposure. There were five exposures.

A total of nine pre-annealed fresh OSLDs was used in order to study the reproducibility of the device response under identical mammography screening conditions. Initially, five dosimeters were placed over a 45-mm mammography phantom at the reference position, as in the calibration experiment, and irradiated with the $32-\mathrm{kVp} 100-\mathrm{mAs}$ X-ray beam with a $\mathrm{Mo} / \mathrm{Rh}$ target/filter combination, as shown in Figure 3. After each exposure, one dosimeter from a fixed position was replaced by a fresh dosimeter. In the process, the four OSLDs, out of five, subsequently received five exposures, and each of the other five dosimeters received a single exposure. After the irradiation, all the OSLDs were read out. The readings of the four dosimeters represent accumulated responses from five consecutive exposures. The sum of the responses of the other five OSLDs is from a single exposure, where each of them was exposed only once.

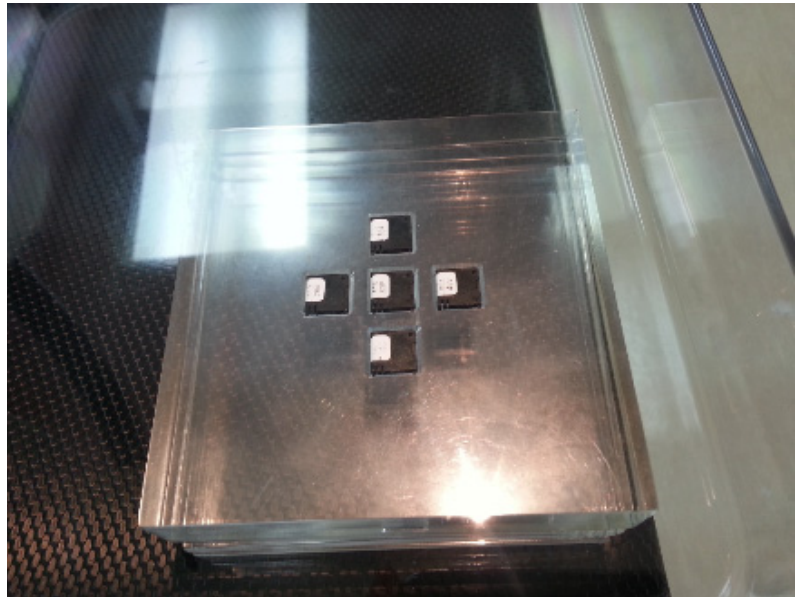

Figure 3. OSLD placement during the reproducibility test.

\subsection{Linearity test}

A set of five pre-annealed fresh OSLDs was placed over the 45-mm mammography phantom, as in the experimental setup shown in Figure 3. Each set of dosimeters was calibrated with various energies $(25,28$ and $32 \mathrm{kVp})$ then irradiated with the same calibration energies and a Mo/Rh target/filter combination. The OSLDs' responses were read out, and the readings were then repeated four times for examination of the signal depletion. The net counts obtained from the OSLDs at different tube potentials were compared with the dose increment, as determined from comparison with the reference dose. The reading of the ionization chamber for the respective beam quality was considered as the reference dose.

\subsection{Angular Dependence}

Sets of pre-annealed fresh OSLDs, with two dosimeters in each set, were placed over the 45-mm mammography phantom (bolus), as shown in Figure 4, and irradiated with a $32-\mathrm{kVp}$ beam with $100-\mathrm{mAs}$ tube loading and a Mo/Rh target/filter combination. The OSLDs were irradiated at angles of $0^{\circ}, 45^{\circ}$, $90^{\circ}, 135^{\circ}, 180^{\circ}$ and $270^{\circ}$. The position with the OSLD serial number directly facing the incident beam was set as the $0^{\circ}$ angular position, and then the OSLD placement was rotated clockwise along the $X-Z$ axis from $0-270^{\circ}$, as illustrated in Figure 5.

\subsection{Breast depth dose distribution}

A set of five pre-annealed fresh OSLDs were used for this test, in an attempt to study their responses at various breast depths under mammography screening conditions. The dosimeters were placed over a 45-mm mammography phantom, taking the skin surface as the $0-\mathrm{cm}$ reference depth, and irradiated with the $32-\mathrm{kVp} 100-\mathrm{mAs} \mathrm{X}$-ray beam with a Mo/Rh target/filter combination. The experimental setup is shown in Figure 6. Under identical exposure setup conditions, the sets of OSLDs were irradiated by placing them at various 


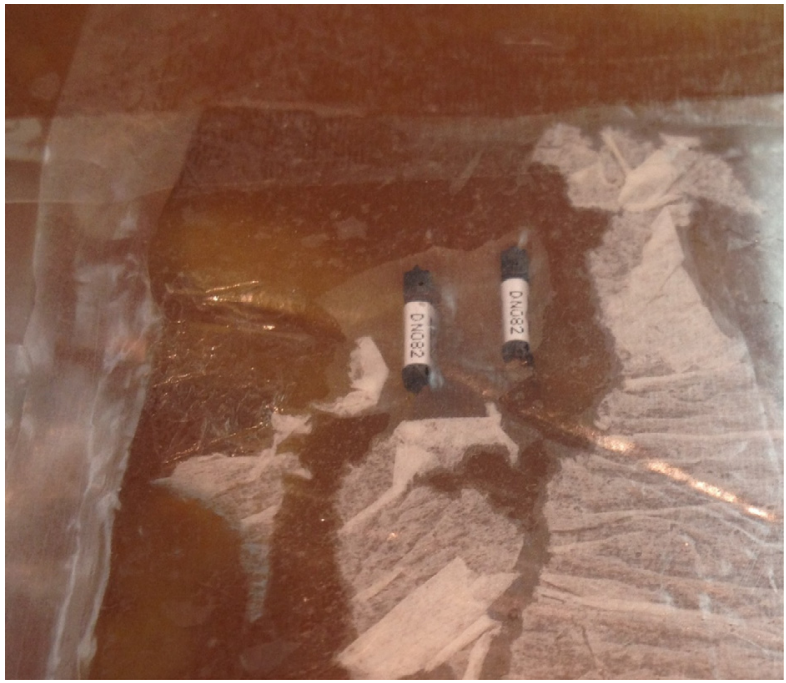

Figure 4. OSLDs at $90^{\circ}$ orientation.

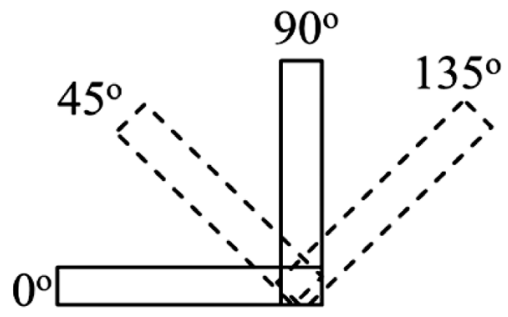

Figure 5. Schematic of OSL orientation along $X-Z$ axis.

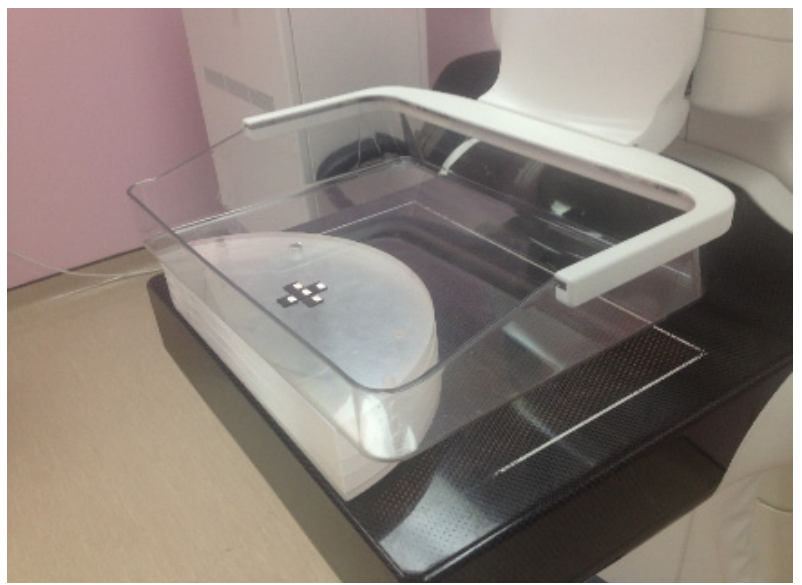

Figure 6. OSLD placement for breast depth dose distribution study.

depths $(0,1,2,3.5$ and $4.5 \mathrm{~cm})$ inside the mammography phantom. The exposed dosimeters were read out as usual, and the reading of each dosimeter was repeated four times in order to examine the signal depletion.

\subsection{Spatial distribution}

One set of five pre-annealed fresh OSLDs was used for this test, in order to study the device responses at various locations in the field of view (FOV) during mammography screening.

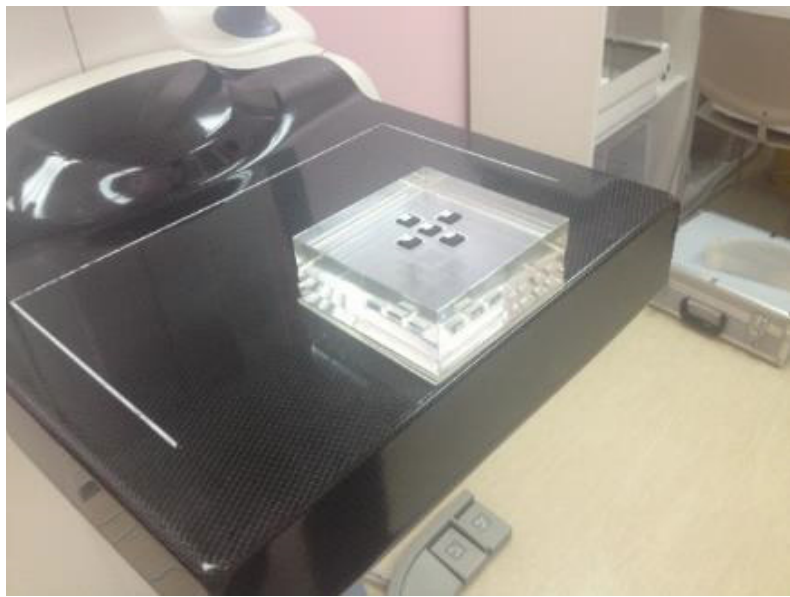

Figure 7. OSLDs $10.5 \mathrm{~cm}$ from right side of FOV.

The OSLDs were placed over a 45-mm mammography phantom at the center $(14.5 \mathrm{~cm})$ of a $29 \times 22 \mathrm{~cm}^{2} \mathrm{FOV}$, and irradiated with the $32-\mathrm{kVp} 100-\mathrm{mAs}$ beam with a Mo/Rh target/filter combination. With the same machine parameter setup and procedures, the OSLD sets were irradiated by placing them at positions $10.5 \mathrm{~cm}$ from the right side of the FOV (Figure 7), $10.5 \mathrm{~cm}$ from the left side of the FOV, and $6 \mathrm{~cm}$ from the chest edge wall. The exposed OSLD responses were then read out.

\subsection{Average glandular dose}

Three sets of five pre-annealed fresh OSLDs were used for measurement of the $K_{i}$ of the target organ, in order to calculate the AGD of the breast glandular tissues. The OSLDs were placed over a 45-mm-thick polymethyl methacrylate (PMMA) mammography phantom, representing $50-\mathrm{mm}$ breast thickness, at distances varying from $40-60 \mathrm{~mm}$ from the chest edge wall, as shown in Figure 6. Each set of OSLDs was irradiated with a different exposure setup, for various combinations of the following parameters: $25-, 28-$ and $32-\mathrm{kVp}$ tube potential, 63-, 71- and 100-mAs tube loading, and Mo/Rh, Rh/Rh and Mo/Mo target/filter combinations, using manual and auto modes. The OSLD responses were read out for each combination. The signal depletion was checked by subsequently reading out the dosimeters four times. The AGD to the breast was calculated using the following expression:

$$
D_{G}=C_{\mathrm{DG} 50, \mathrm{Ki}, \mathrm{PMMA}} \times s \times k_{i},
$$

where $D_{G}$ (mGy) represents the dose to glandular tissue $(\mathrm{mGy} / \mathrm{mGy}), C_{\mathrm{DG} 50, \mathrm{Ki}, \mathrm{PMMA}}$ is a correction factor (about $10 \%$ ) that was applied as a conversion coefficient, calculated from the HVL, which is used to calculate the mean glandular dose to a $50-\mathrm{mm}$ standard breast of $50 \%$ glandularity from the incident air kerma $\left(k_{i}\right)$ for a $45-\mathrm{mm}$ PMMA phantom. This coefficient combines the coefficient $C_{D_{G 50}, K_{i}}$ (the conversion coefficient for calculating the mean glandular dose for a breast of $50 \%$ glandularity) and the ratio of the values of the incident air kerma for the standard breast and the standard phantom. This coefficient converts the incident air kerma at the entrance surface of the PMMA phantom to the mean glandular dose for the 
Table 1. OSL response against ionization chamber readings.

\begin{tabular}{ccccccccc}
\hline $\begin{array}{c}\text { PMT } \\
\text { counts } \\
\text { (Net) }\end{array}$ & Average & $\begin{array}{c}\text { Ionization } \\
\text { chamber } \\
\text { reading } \\
\text { (mrad) }\end{array}$ & Average & $\begin{array}{c}\text { Calibration } \\
\text { Factor }(\mathbf{C F}) \\
\text { (counts/mrad) }\end{array}$ & $\begin{array}{c}\text { Average } \\
\text { CF } \\
\text { (counts/mrad) }\end{array}$ & $\begin{array}{c}\text { \%Std. } \\
\text { Dev }\end{array}$ & $\begin{array}{c}\text { Converted } \\
\text { Average } \\
\text { CF }\end{array}$ & $\begin{array}{c}\text { \%Std. } \\
\text { Dev }\end{array}$ \\
\hline 24290 & 24846 & 956.5 & 956.4 & 30.972 & 31.681 & $1.96 \%$ & 3168 & $1.96 \%$ \\
25060 & & 956.4 & & 31.954 & & & & \\
\hline 25188 & & 956.3 & & 32.117 & & & & \\
\hline
\end{tabular}

standard breast. Furthermore, $s$ is the spectral correction factor for the selected target/filter combination (3). The following expression was used for the calculation of $K_{i}$ :

$$
K_{i}=\frac{\text { Net Dose }(m G y)}{B},
$$

where Net Dose $(m G y)$ is the value calculated from the OSL response and $B$ is the backscatter correction factor. The values of $C_{\text {DG50,Ki,PMMA }} B$ and $s$ were taken from IAEA (2007). Wherever necessary, the $C_{\mathrm{DG} 50, \mathrm{Ki} \text {,PMMA }}$ and $B$ values were interpolated.

\section{Results}

\subsection{Calibration}

The exposed OSL responses (PMT counts), together with the ionization chamber exposure readings, are presented in Table 1 . The necessary corrections for the OSLD batch sensitivity were applied. The converted calculated calibration factor was 3168 counts $\mathrm{mGy}^{-1}$ with $1.96 \%$ standard deviation.

\subsection{Reproducibility}

Figure 8 shows the OSL nanoDot dosimeter response accumulated from five consecutive exposures, and the sum of the single-exposure values of each of the five other OSLDs. The responses of the set of OSLDs were reproducible within $\pm 0.6 \%$. The reproducibility of the responses of five dosimeters that received a single exposure are also presented (Figure $8 \mathrm{~b}$ ).

\subsection{Linearity}

The linearity of the OSLD responses for exposures with different tube potential $(25,28$ and $32 \mathrm{kVp})$ and tube loading (40, 60, 80 and $100 \mathrm{mAs})$ values is shown in Figure 9.

Figure 9 represents the OSLD response for each tube loading value with respect to the increment in mAs. Good linearity can be seen, with $R^{2}>0.99$. To analyze the linearity of the OSLD response in relation to the reference doses, the fitting equation from each tube loading from Figure 9 was used. This is shown in Figure 10, which presents the net counts of the OSL nanoDot dosimeters (the amount of photons captured by the PMT) for each tube loading value versus reference doses from the mammography ion chamber readings. (a)

REPRODUCIBILITY TEST OF OSLD RESPONSE (ACCUMULATED) VS SUM 5 SINGLE OSLD RESPONSE

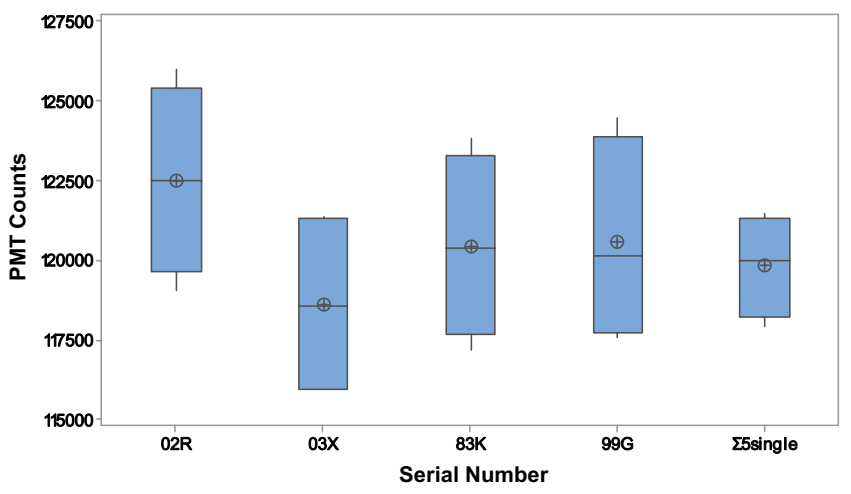

(b) REPRODUCIBILITY TEST OF OSLD RESPONSE (SINGLE)

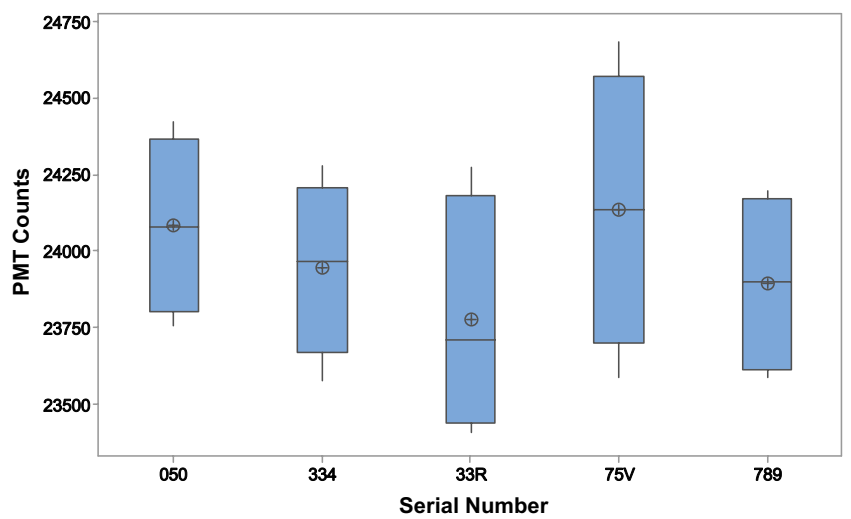

Figure 8. (a) Reproducibility test of OSLD response (Accumulated) vs. Sum of 5 OSL Response. (b) Reproducibility test of OSLD response (Single).

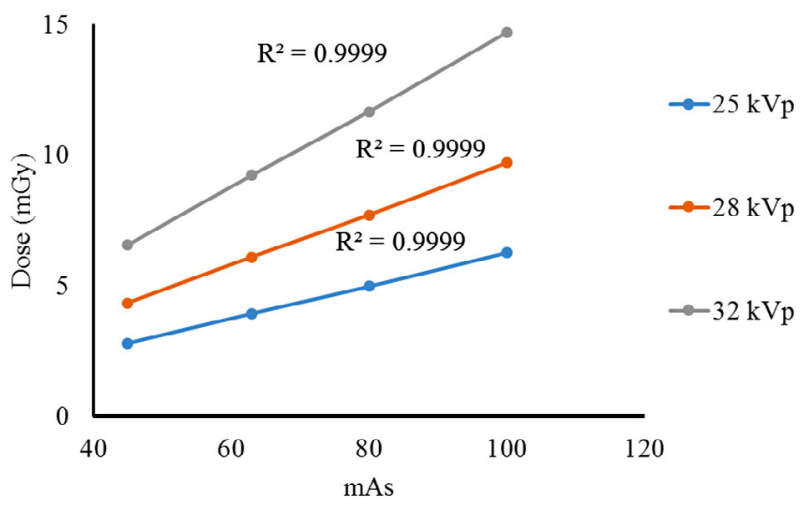

Figure 9. Linearity of OSLD response. 


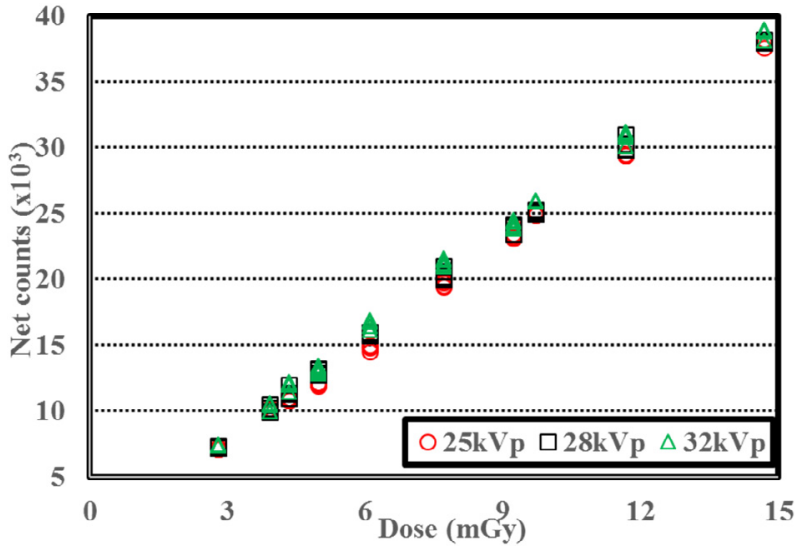

Figure 10. Linearity of OSLD response to dose.

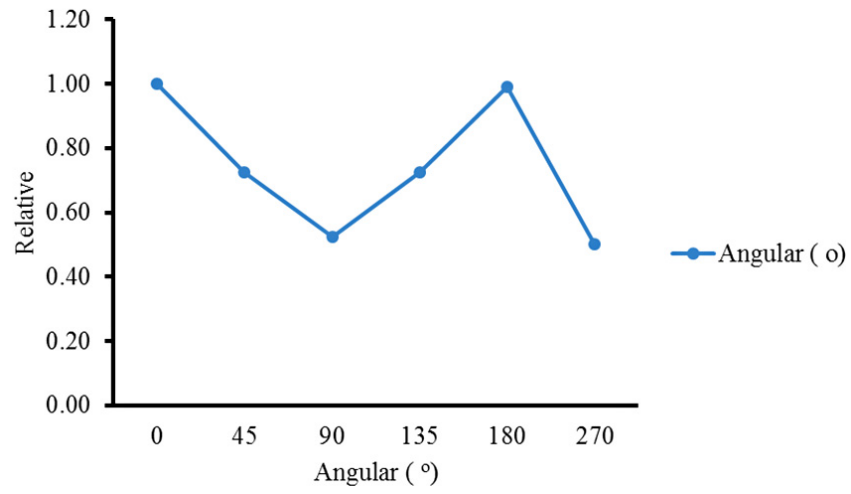

Figure 11. OSLD response for different orientations.

Good linearity between each nanoDot response with the ion chamber readings (reference dose) was shown, with all $R^{2}$ values greater than $0.99(p<0.05)$. The difference between the OSLD responses for each tube loading was found to be less than $2 \%$ on average. However, some energy dependence, up to $5 \%$, was observed in this tube loading range $(25-32 \mathrm{kVp})$, especially when the incident air kerma was higher than $6 \mathrm{mGy}$.

\subsection{Angular dependence}

The angular dependence of the OSLD response was examined for a $32-\mathrm{kVp} 100-\mathrm{mAs} \mathrm{Mo} / \mathrm{Rh}$ target/filter beam. Doses measured from angled nanoDots were normalized to the value at the $0^{\circ}$ angular position, when the dosimeter serial number was oriented toward the incident beam. Thus, the $180^{\circ}$ orientation corresponds to the position at which the opposite side (barcode) of the OSLD was facing the beam. The angular dependence response results are shown in Figure 11.

\subsection{Breast depth dose distribution}

The absorbed doses in the breast were measured at varying depths for a $32-\mathrm{kVp} 100-\mathrm{mAs} \mathrm{Mo} / \mathrm{Rh}$ beam. The assessed doses were normalized to the dose obtained at $0-\mathrm{cm}$ depth (skin dose). Figure 12 shows the depth dose distribution to the breast.

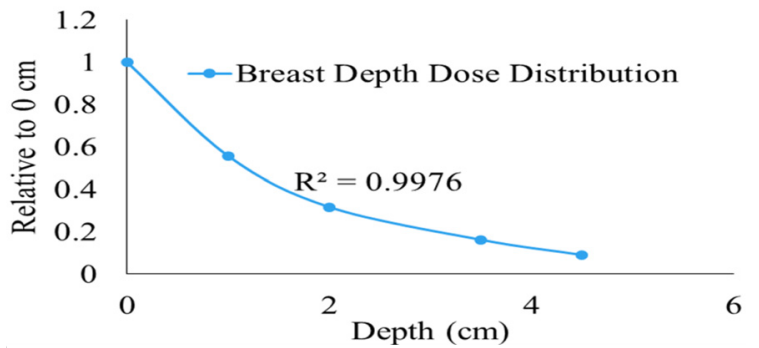

Figure 12. OSLD response in breast depth dose distribution study.

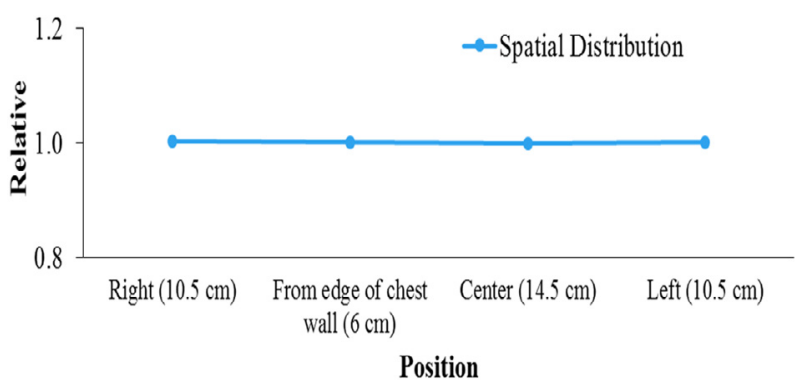

Figure 13. Lateral distribution over breast phantom of OSLD response.

\subsection{Spatial distribution}

Sets of OSLDs were placed laterally on the PMMA breast phantom at equal distances from the edge of the chest wall, and exposed to the $32-\mathrm{kVp} 100-\mathrm{mAs}$ beam with a $\mathrm{Mo} / \mathrm{Rh}$ target/filter combination. The lateral distribution of the OSLD response normalized with that obtained $6 \mathrm{~cm}$ from the edge of chest wall is shown in Figure 13.

\subsection{Average glandular dose}

The AGD was calculated from varying $K_{i}$ values at different beam energies, beam currents, target/filter combinations, distances from the chest edge wall and exposure modes. The calculated AGD values are shown in Table 2.

\section{Discussion}

The generated calibration factor for the measurement scenario was 3168 counts $\mathrm{mGy}^{-1}$ with $1.94 \%$ standard deviation with $0.5 \% \pm 0.3 \%$ signal depletion for each reading. According to Figure $8 \mathrm{~b}$, the difference among the responses of the five single OSLDs was within $\pm 1.44 \%$. Furthermore, the difference between each accumulated OSLD response from five consecutive exposures was within $\pm 2.55 \%$. When the average of accumulated OSLD responses was compared with the sum of five single-exposure OSLD responses, the difference was found to be only $\pm 0.6 \%$ (Figure $8 \mathrm{a}$ ). Thus, accumulated doses from multiple exposures did not affect the net results with respect to the dose from a single exposure. This shows the stability of the OSLD responses under multi-exposure conditions, and for breast dosimetry for mammography screening conditions. Good linearity of the OSL nanoDot dosimeter responses with 
Table 2. Calculated AGD values for different beam properties.

\begin{tabular}{|c|c|c|c|c|c|c|c|}
\hline Mode & $k V p$ & mAs & Target/filter & PMT counts & $K_{i}(\mathrm{mGy})$ & AGD (mGy) & $\begin{array}{c}\text { Remarks } \\
(\mathrm{cm} \text { from chest edge) }\end{array}$ \\
\hline \multirow[t]{15}{*}{ Manual } & 25 & 100 & $\mathrm{Mo} / \mathrm{Rh}$ & $16902 \pm 2.42 \%$ & 6.51 & $1.39 \pm 0.111$ & 5 \\
\hline & 28 & 63 & $\mathrm{Mo} / \mathrm{Rh}$ & $15176 \pm 1.57 \%$ & 5.620 & $1.312 \pm 0.105$ & 4.7 \\
\hline & & 71 & Mo/Mo & $20432 \pm 0.56 \%$ & 7.646 & $1.561 \pm 0.125$ & 4 \\
\hline & & & $\mathrm{Mo} / \mathrm{Rh}$ & $17481 \pm 0.96 \%$ & 6.475 & $1.511 \pm 0.121$ & 4 \\
\hline & & & & $17103 \pm 1.5 \%$ & 6.34 & $1.479 \pm 0.118$ & 4.7 \\
\hline & & & & $17657 \pm 2.2 \%$ & 6.335 & $1.479 \pm 0.118$ & 6 \\
\hline & & & $\mathrm{Rh} / \mathrm{Rh}$ & $15874 \pm 1.5 \%$ & 5.873 & $1.449 \pm 0.116$ & 4 \\
\hline & & 100 & $\mathrm{Mo} / \mathrm{Rh}$ & $24570 \pm 2.2 \%$ & 9.1 & $2.123 \pm 0.170$ & 4 \\
\hline & & & $\mathrm{Mo} / \mathrm{Rh}$ & $25284 \pm 1.7 \%$ & 9.73 & $2.08 \pm 0.166$ & 5 \\
\hline & 32 & 71 & $\mathrm{Mo} / \mathrm{Rh}$ & $26391 \pm 1.3 \%$ & 9.716 & $2.431 \pm 0.194$ & 4 \\
\hline & & & $\mathrm{Rh} / \mathrm{Rh}$ & $24804 \pm 1.2 \%$ & 9.095 & $2.496 \pm 0.200$ & 4 \\
\hline & & 100 & $\mathrm{Mo} / \mathrm{Rh}$ & $38098 \pm 0.8 \%$ & 14.026 & $3.509 \pm 0.281$ & 4 \\
\hline & & & & $37602 \pm 0.4 \%$ & 13.843 & $3.463 \pm 0.277$ & 4.7 \\
\hline & & & & $39006 \pm 1.88 \%$ & 15.01 & $3.42 \pm 0.274$ & 5 \\
\hline & & & & $38388 \pm 1.7 \%$ & 14.135 & $3.536 \pm 0.283$ & 6 \\
\hline \multirow[t]{5}{*}{ Auto } & 28 & 73.8 & $\mathrm{Mo} / \mathrm{Rh}$ & $19313 \pm 2.37 \%$ & 7.153 & $1.669 \pm 0.134$ & 4 \\
\hline & & & $\mathrm{Mo} / \mathrm{Rh}$ & $19567 \pm 2.29 \%$ & 7.53 & $1.61 \pm 0.129$ & 5 \\
\hline & & & $\mathrm{Mo} / \mathrm{Rh}$ & $19566 \pm 1.71 \%$ & 7.53 & $1.61 \pm 0.129$ & 5 \\
\hline & & & $\mathrm{Mo} / \mathrm{Rh}$ & $18914 \pm 2.87 \%$ & 7.005 & $1.635 \pm 0.131$ & 6 \\
\hline & & 74.1 & $\mathrm{Mo} / \mathrm{Rh}$ & $18852 \pm 1.2 \%$ & 6.964 & $1.624 \pm 0.130$ & 4 \\
\hline
\end{tabular}

different beam energies was also observed, as shown in Figure 9. The linearity test resulted in $\mathrm{R}^{2}$ values greater than 0.99 , which is in conformity with the findings of previous works (IAEA, 2007; Jursinic, 2007; Perks et al., 2007; Yukihara et al., 2008; Al-Senan et al., 2011; Yusuf et al., 2014). Due to the good linearity of response between each energy, the relative response between each energy could be determined and used for measuring the dose without repeating the calibration. In addition, the energy dependence was found to be less than $2 \%$ in the $25-32 \mathrm{kVp}$ range, and increased when the incident air kerma was higher than $6 \mathrm{mGy}$, as shown in Figure 10. This energy dependence variation was primarily due to the small variation in the beam energy range selected for clinical screening, while the photon interaction increased with the increased dose; still, about 5\% difference was found during this experiment (Jursinic, 2007; Yukihara et al., 2008; Al-Senan et al., 2011). Due to the small energy dependence of the response in the energy range of $25-32 \mathrm{kVp}$, calibration at each energy was avoided. However, an energy dependence correction factor should be considered for higher precision measurement.

The angular dependence test exhibited the most noticeable deviation for the $90^{\circ}$ and $270^{\circ}$ orientations, at which the dose decreased by 48-50\% (Figure 11). However, the measured doses at the $0^{\circ}$ and $180^{\circ}$ angles exhibited deviations of only $\sim 1 \%$ from the data reported in the literature (Boone et al., 2001; Jursinic, 2010). This is because the flat surface OSLDs are directly exposed to the X-ray beam under these conditions. On the other hand, for OSLDs placed at other angles, less surface area is exposed to the X-ray beam, resulting in a lower exposure. The decrease in the dose based on the OSLD orientations observed in the present work is within the range of the values reported in the literature (IAEA, 2001; Oliveira et al., 2014). It ensures that, when OSLDs are used for monitoring purposes, the results are not dependent on exposure from the front or back sides (barcode facing the beam). While using OSLDs in breast dosimetry, the utmost care must be taken in positioning the dosimeter because of its high angular dependence.

The absorbed doses to the breast at varying depths were measured with the 32-kVp 100-mAs Mo/Rh beam. The measured dose at $1-\mathrm{cm}$ depth was found to be $56 \%$ of the entrance surface air kerma and, at $2 \mathrm{~cm}$ depth, the absorbed dose to breast tissue was $32 \%$ of the entrance surface air kerma (refer to Figure 12). The breast dose was found to decrease with increasing breast depth, and the $\mathrm{R}^{2}$ value was almost unity. A similar trend has been observed in other studies (Jursinic, 2010; Tsai et al., 2010). The measured $K_{i}$ values at different lateral positions were found to be almost equal, with a maximum deviation of $6 \%$. Figure 13 yields $1.001 \pm 0.09 \%$ as the value for the average relative response of the OSL nanoDots at the reference point (center) with respect to other lateral positions. The calculated AGD values for a $45-\mathrm{mm}$ compressed-breast-thickness phantom are in the 1.3-3.5-mGy range, depending on the tube voltage, beam current and target/filter combinations. The respective value of $K_{i}$ lies in the 5.6-14.1 $\mathrm{mGy}$ range, depending on the beam energy, $\mathrm{mAs}$ and target/filter combinations, in agreement with previous studies (Aznar et al., 2005; Edwards et al., 2005; Huang et al., 2008; Tsai et al., 2010; Lavoie et al., 2011; Chen et al., 2012). Using the auto-exposure control (AEC) mode and a $28-\mathrm{kVp}$ 73.8-mAs $\mathrm{Mo} / \mathrm{Rh}$-target-filter combination, we verified the reproducibility of the AGD values for a 45-mm PMMA breast 
phantom (representative of 50-mm breast thickness), by taking measurements for five exposures under identical geometries (refer to the data shown in Table 2). The calculated AGDs were found to be reproducible within $\pm 1.5 \%$.

\section{Conclusion}

In the present study, the OSLD responses exhibited good reproducibility, linearity, angular dependence, breast depth dose distribution performance and spatial distribution uniformity in mammography screening. The OSLD responses were found to be stable under identical multiple exposure conditions. Further, the variation of the energy dependence response was found to be less than $2 \%$ in the $25-32-\mathrm{kV}$ tube potential range when the incident air kerma was below $6 \mathrm{mGy}$. By using different tube loading and target/filter combinations, we verified that the average glandular dose (AGD) value for a breast is linear with increased energy ( $\mathrm{kVp}$ and $\mathrm{mAs})$, and depends on the target/filter combination used in the breast screening of a particular patient. Higher energy and a high beam current impart a higher absorbed dose to the breast glandular tissue. The following exposure-setting combination may be preferred for mammography screening of a 50-mm breast thickness (45-mm compressed breast thickness) in auto control mode of the GE Mammograph Senographe Essential at KAUH: 28-kVp tube potential, 73.8-mAs tube loading and a Mo/Rh target/filter combination. It is concluded that the OSL nanoDot dosimeter may be considered as a potential candidate for use in quality control programs relevant to mammography screening.

Acknowledgements. This project was supported by the NSTIP Strategic Technologies Program in the Kingdom of Saudi Arabia - Award No. (08-MED113-03). The authors also acknowledge with thanks the Science and Technology Unit, King Abdulaziz University, for technical support.

\section{References}

Al-Senan R.M. et al. (2011) Characteristics of an OSLD in the diagnostic energy range, Med. Phys. 38, 4396-4405.

Andersen C.E. et al. (2009) Characterization of a fiber-coupled $\mathrm{Al}_{2} \mathrm{O}_{3}: \mathrm{C}$ luminescence dosimetry system for online in vivo dose verification during ${ }^{192}$ Ir brachytherapy, Med. Phys. 36, 708-718.

Andersson I. (1986) Mammography in clinical practice. Eastman Kodak Company, Rochester.

Aznar M.C. et al. (2005) A Monte Carlo study of the energy dependence of $\mathrm{Al}_{2} \mathrm{O}_{3}: \mathrm{C}$ crystals for the real-time in vivo dosimetry in mammography, Radiat. Prot. Dosim. 114, 444-449.

Boone J.M. et al. (2001) Dedicated breast CT: radiation dose and image quality evaluation, Radiology 221, 657-667.

Chen B. et al. (2012) Analysis of patient dose in full field digital mammography, Eur. J. Radiol. 81, 868-872.
Dance D.R. (1990) Monte-carlo calculation of conversion factors for the estimation of mean glandular breast dose, Phys. Med. Biol. 35, 1211-1219.

Dance D.R. et al. (1999) Breast dosimetry, Appl. Radiat. Isotopes 50, 185-203.

Dance D.R. et al. (2000) Additional factors for the estimation of mean glandular breast dose using the UK mammography dosimetry protocol, Phys. Med. Biol. 45, 3225-3240.

Edwards C.R. et al. (2005) The low energy X-ray response of the $\mathrm{LiF}: \mathrm{Mg}: \mathrm{Cu}: \mathrm{P}$ thermoluminescent dosemeter: a comparison with LiF:Mg:Ti, Br. J. Radiol. 78, 543-547.

US Dpt of Health and Human Services, Bassett L.W. (1995) High quality mammography: information for referring providers, Breast J. 1, 331-332.

Huang S. et al. (2008) The effect of skin thickness determined using breast CT on mammographic dosimetry, Med. Phys. 35, 11991207.

IAEA (2007) Dosimetry in diagnostic radiology: an international code practice, Technical reports series no. 457. IAEA, Vienna, pp. $155-187$.

Jursinic P.A. (2007) Characterization of optically stimulated luminescent dosimeters, OSLDs, for clinical dosimetric measurements, Med. Phys. 34, 4594-4604.

Jursinic P.A. (2010) Changes in optically stimulated luminescent dosimeter (OSLD) dosimetric characteristics with accumulated dose, Med. Phys. 37, 132-140.

Lavoie L. et al. (2011) Characterization of a commercially-available, optically-stimulated luminescent dosimetry system for use in computed tomography, Health Phys. 101, 299-310.

Oliveira B.B. et al. (2014) Mean glandular dose for different angles of the X-ray tube using different glandularity phantoms, Radiat. Phys. Chem. 95, 202-204.

Oliveira M. et al. (2007) Average glandular dose and phantom image quality in mammography, Nucl. Instrum. Methods Phys. Res. A 580, 574-577.

Perks C.A. et al. (2007) Medical dosimetry using Optically Stimulated Luminescence dots and microStar readers. In: Proceedings of the 12th Congress of the International Radiation Protection Association (IRPA12), pp. 1-10. IAEA.

Poliei S.R. et al. (1987) Occult breast cancer; prevalence and radiographic detectability, Radiology 163, 459-462.

Tsai H.Y. et al. (2010) Evaluation of depth dose and glandular dose for digital mammography, Radiat. Meas. 45, 726-728.

Viamonte L.A.R. et al. (2008) Radiotherapy dosimetry using a commercial OSL system, Med. Phys. 35, 1261-1266.

Vinnicombe S. et al. (2009) Full-field digital versus screen-film mammography: comparison within the U.K. breast screening program and systematic review of published data, Radiology 251, 347358.

Young K.C. (2002) Radiation doses in UK trial of breast screening in women aged 40-48 years, Br. J. Radiol. 75, 362-370.

Yukihara E.G. et al. (2008) Optically stimulated luminescence (OSL) dosimetry in medicine, Phys. Med. Biol. 53, R351-R379.

Yusuf M. et al. (2014) Characterization of the Optically Stimulated Luminescence nanoDot for CT Dosimetry, Life Sci. J. 11, 445450.

Zoetelief J. et al. (1996) European protocol on dosimetry in mammography, Radiat. Prot. Dosim. 80, 191-193.

Cite this article as: N. Alothmany, N.I. Molla, M. Yusuf, A. Saoudi, N. Mail, D. Alothmany, M.A. Khafaji, H. Natto, M. Tayeb, F. Nadwi, A. Jiman, A.A. Kinsara. Characterization of optically stimulated luminescence for assessment of breast doses in mammography screening. Radioprotection 51(1), 51-58 (2016). 